Journal of Pediatric Gastroenterology and Nutrition, Publish Ahead of Print

DOI : 10.1097/MPG.0000000000002811

\title{
Maternal microchimerism in cord blood and risk of celiac disease in childhood
}

German Tapia*1 ${ }^{1}$ Ph.D, Georgina Mortimer*², BSc, Jody Ye $^{2}, \mathrm{PhD}$,

Karl Mårild ${ }^{3}, \mathrm{MD}, \mathrm{PhD}$, Saranna Chipper-Keating ${ }^{2}$, MSc,

Benjamin T. Gillard ${ }^{2}$, MSc, Marte K. Viken ${ }^{4}, \mathrm{PhD}$, Benedicte A. Lie ${ }^{4}, \mathrm{PhD}$,

Lars C. Stene ${ }^{1}$, PhD, Kathleen M. Gillespie ${ }^{2}$, PhD, Ketil Størdal ${ }^{1,5}$, MD, PhD.

\section{* Shared first authorship}

\section{Affiliations:}

${ }^{1}$ Norwegian Institute of Public Health, Oslo, Norway

${ }^{2}$ Diabetes and Metabolism, Bristol Medical School, University of Bristol, Bristol, UK

${ }^{3}$ Department of Pediatrics, Institute of Clinical Sciences, The Sahlgrenska Academy at

University of Gothenburg and Queen Silvia Children's Hospital, Gothenburg, Sweden

${ }^{4}$ Department of Immunology, Rikshospitalet, Oslo University Hospital, Oslo, Norway and

Department of Medical Genetics, University of Oslo, Oslo, Norway

${ }^{5}$ Pediatric Department, Østfold Hospital Trust, Grålum, Norway

Corresponding author: German Tapia, Department of Chronic Diseases and Ageing, Norwegian Institute of Public Health, P.O. Box 222 Skøyen, NO-0213 Oslo, Norway. Telephone: 004721078410. E-mail: german.tapia@fhi.no 
Running short title: Maternal microchimerism in cord blood and CD

\section{Conflicts of interest and Source of Funding:}

Dr. Størdal was supported by an unrestricted grant from the Oak Foundation, Geneva, Switzerland. Costs of all data acquisition, including laboratory assays in MoBa (the sub-study PAGE; Prediction of Autoimmune Diabetes and Celiac Disease in Childhood by Genes and Perinatal Environment), was supported by grant 2210909/F20 from the Norwegian Research Council (Dr. Stene). No conflict of interest has been declared, and the authors alone are responsible for the content and writing of the paper.

\section{Abstract}

Objectives: During pregnancy, small quantities of maternal cells are naturally transmitted to the fetus. This transmission, termed maternal microchimerism (MMc), has been implicated in autoimmune diseases but its potential role is unclear. We aimed to investigate if MMc at birth predicted childhood celiac disease (CD) risk, a common immune-mediated enteropathy often presenting in childhood.

Methods: We designed a case-control study, nested in the Norwegian Mother, Father and Child Cohort. Participants were HLA class II typed to determine non-inherited, non-shared maternal alleles (NIMA). Droplet digital (dd) PCR assays specific for common HLA class II NIMAs (HLA-DQB1*03:01, *04:02 and *06:02/03) were used to estimate the quantity of maternal DNA, as a marker of maternal cells, in cord blood DNA from 124 children who later developed clinically diagnosed CD (median age at end of study 7.4 years, range 3.6-12.9) and 124 random controls. We tested whether presence of MMc was associated with $\mathrm{CD}$ using logistic regression, and compared ranks between cases and controls. 
Results: MMc, e.g. maternal HLA antigens not inherited by the child, was found in $42 \%$ of cases and $43 \%$ of controls, and not associated with CD (odds ratio (OR) $0.97,95 \%$ confidence interval [CI] 0.58-1.60). The ranks of MMc quantities in cases and controls were also similar (MannWhitney $\mathrm{U}$ test $\mathrm{p}=0.71$ ). The subgroup with $H L A-D Q B 1: 03 * 01$ as their NIMA had a potential association with MMc, where levels >median was associated with CD (OR 3.78, 95\%CI 1.2811.18).

Conclusions: MMc measured in cord blood was not associated with later risk of CD.

Keywords: Microchimerism, HLA, Celiac Disease, Pregnancy, Childhood, mother and child cohort study

\section{What is known}

- Maternal microchimerism is the naturally occurring transmission of a small number of maternal cells to the fetus during pregnancy.

- Maternal microchimerism has been linked to some autoimmune diseases, but it is unclear how it is involved in disease development or tissue regeneration.

- Celiac disease is an increasingly prevalent disease but there are no studies on a possible association with maternal microchimerism.

\section{What is new}

- The presence, or levels of, maternal microchimerism at birth were not associated with later celiac disease in the offspring. 


\section{Introduction}

Maternal microchimerism $(\mathrm{MMc})$ results from the transfer of maternal cells to the fetus during pregnancy. Maternal cells from lymphoid and myeloid cell lineages, as well as hematopoietic progenitors, are present in offspring tissues and can persist to adulthood, $(1,2)$ suggesting a state of immunological tolerance to non-inherited maternal antigens (NIMA). Protection of maternal cells from the developing fetal immune response is through induction of regulatory $\mathrm{T}$ cells $\left(\mathrm{T}_{\mathrm{reg}}\right)$.

(3) Mice studies have confirmed that MMc induces stable tolerance to NIMA in offspring. Female offspring with mates sharing these antigens experience reduced fetal wasting, establishing a role for MMc in reproductive fitness. (4) Maternal antigens during pregnancy represents the first fetal immunological challenge and could influence tolerance to antigens encountered in utero, and possibly after birth. In mice, T cell MMc from alloreactive mothers resulted in inflammation and diminished frequency of $\mathrm{T}_{\text {regs }}$ in offspring, which indirectly links MMc and autoimmunity. (5) Several immune-mediated diseases, including type 1 diabetes (T1D), have been associated with increased levels of MMc measured after disease onset, (6-11) but it is unclear whether MMc levels at birth predict future disease development. While maternal cells may expand postnatally, possibly as a result of disease, identification of differential levels already at birth could provide improved insight into potential mechanisms and potentially aid in disease prediction. To the best of our knowledge, the only previous study of cord blood MMc and later immune-mediated disease is our own study of T1D, a disease that shares etiological traits with CD. We hypothesized that a higher degree of MMc would be associated with lower risk of both CD and T1D in The Norwegian Mother, Father and Child Cohort Study (MoBa). We have recently published our results on T1D, where we did not find any clear relationship. (12) 
Although MMc in cord blood was not associated with later development of T1D, MMc could play a role in $\mathrm{CD}$.

Celiac disease (CD) is a chronic immune-mediated disorder where dietary gluten causes an inflammatory response leading to villous atrophy and intestinal malabsorption. CD and T1D share genetic factors, such as high-risk Human Leucocyte Antigen (HLA) genotypes, and could share pathogenic mechanisms. $(13,14)$ The diseases also likely have non-shared factors and biological mechanism. HLA alleles confer genetic risk, with almost all CD patients expressing HLA-DQ2.5, HLA-DQ8 or HLA-DQ2.2. (15) ) While the exact mechanism linking MMc to autoimmunity has not been delineated in detail, it is conceivable that MMc can influence tolerance to tissue transglutaminase, gluten peptides, or both, by a general influence on immunological tolerance. Other possibilities includes a potential role in tissue healing capacity, or antiviral response, by proliferation and differentiation of maternal cells as a response to injury.(16) CD specific autoantibodies usually often develop the first years of life early in life. $(17,18)$ The unknown processes leading to CD development could start in fetal life, (19) and could possibly be associated to MMc (e.g development of a tolerogenic or proinflammatory phenotype in the fetus, or MMc could be a marker for a healthy pregnancy).

In this study, we aimed to investigate whether quantity of maternal cells, estimated by genomic equivalents of maternal DNA in cord blood at birth, were associated with later risk of CD.

\section{Methods}

We designed a nested case-control study in the Norwegian Mother, Father and Child Cohort Study (MoBa) (20), which recruited approximately 114,000 children and their mothers (41\% of eligible mothers participated) from all over Norway during 1999-2008. The current study uses 
data from repeated questionnaires (using the version VIII of the MoBa data), maternal postpartum and offspring cord blood samples (21). All study participants gave written informed consent. The study also uses data (maternal parity, sex, age and cesarean section) from the Medical Birth Registry of Norway (MBRN), which is a national health registry containing information about all births in Norway. The establishment of MoBa and initial data collection was based on a license from the Norwegian Data protection agency and approval from The Regional Committee for Medical Research Ethics. The MoBa cohort is currently regulated by the Norwegian Health Registry Act. The current study was approved by The Regional Committees for Medical and Health Research Ethics.

Ascertainment of case status

For a detailed description of the case status ascertainment, please see our previous publication. (22) Briefly, we used the Norwegian Patient Register (NPR) and parental questionnaires to identify children in MoBa diagnosed with $\mathrm{CD}$, which was validated by contacting a subset of families with children registered with CD in the NPR and asking these to provide details of the diagnostic process. We defined CD as minimum two registrations of the International Classification of Diseases-10 code K90.0 in the NPR by December 31, 2013 or CD reported by parental questionnaires administered when the child is 7 to 8 years. Children with a single entry in the NPR and no confirmation of CD diagnosis in the questionnaires were excluded to reduce the possibility of misclassification. Pediatric celiac disease diagnostic work-up is mainly hospital-based in Norway. Reporting to the NPR is mandatory and linked to governmental reimbursement for funding health services. Parents validated $92 \%$ of the CD diagnoses, with most based on biopsy (83\%) or positive serology. (23) We selected children in the MoBa study that were alive at 1 year of age $(n=113,053)$. From these, we retrieved biobanked blood samples 
from CD cases diagnosed by 1st of January 2013 (blood samples available for 416 of 635 cases). Controls were randomly selected from MoBa participants with available blood samples. Controls were selected randomly from MoBa participants (1009 random dyads, with 550 having available cord blood and 530 having a maternal pregnancy blood sample). As controls planned to be used as shared controls for several studies they were randomly picked. No postnatal blood samples were available to screen for transglutaminase 2 antibodies, nor were controls matched for any characteristic. Random controls identified with diagnosed CD at the end of follow-up were excluded as controls, according to the traditional case-control design for valid estimation of odds ratios for association. (24) Baseline characteristics for those with available blood samples were largely similar to the whole MoBa cohort, except a lower proportion of caesarean section and premature birth (see reference (25)).

After genotyping, we had 916 dyads that were evaluated for MMc. Not all participants had informative NIMA, and ddPCR assays were not available for all HLA alleles, resulting in 124 mother/child-pairs where the child developed CD and 124 control pairs in the analysis. Figure 1B shows a Sankey diagram, where the width of the branches correspond to the number of participants, which show the number of participants at each stage of this study. Data from the same random controls were used in a previous publication. (12) Characteristics of the participants are shown in Table 1.

\section{Sampling}

Maternal venous blood samples were collected in EDTA tubes during pregnancy. At birth, cord blood was obtained from the umbilical cord vein using a syringe $(20,21,26)$. DNA was extracted and stored at the biobank at $-20^{\circ} \mathrm{C}$ until analysis. For details, see $(21,26,27)$. 


\section{Genotyping of human leukocyte antigen}

Mothers and children were genotyped using a custom Illumina Golden Gate assay (Illumina, San Diego, CA). Briefly, tag SNPs $(n=144)$ on chromosome 6 were used to impute human leukocyte antigen (HLA) class II genotype. (28) For each dyad, we inferred the non-inherited, non-shared HLA-DQB1 allele (Figure 1), which was subsequently confirmed by classical HLA genotyping using allele specific PCR on all samples. (29) DNA extraction, genotyping methods and quality control procedures are described in detail in a previous publication (30).

\section{Quantification of maternal microchimerism}

In this study, MMc was defined by the presence of non-inherited, non-shared maternal HLA alleles. Dyads with a non-inherited, non-shared maternal HLA alleles were termed informative and included in the study. For example, if the mother is HLA DQB1 03:01/04:02 and the child is HLA DQB1 04:02 homozygous, the non-inherited non-shared HLA DQB1 is 03:01, which can be measured with our approach (Figure 1). Shared, non-inherited HLA alleles, present if mother is HLA-DQB1 homozygous or if mother and child shares both HLA-DQB1 alleles, would mask the non-inherited maternal allele. For example, if the mother was homozygous HLA-DQB1 03:01 the child would carry at least one HLA-DQB1 03:01 genotype which would make it impossible to quantify the non-inherited maternal DQB1 allele. We termed these noninformative, as we could not separate signals from the MMc from the child`s own HLA-DQB1 genotype, and they were not included in the study (Figure 1). MMc levels were measured by quantifying the amount of NIMA specific DNA in cord blood in informative dyads. We used available, allele-specific droplet digital [dd] ddPCR assays for three common NIMAs (DQB1*03:01, DQB1*04:02, DQB1*06:02/*06:03), as described previously (12) using the ddPCR QX200 system (Bio-Rad, California, USA). The allele-specific ddPCR assays used were 
developed at the time of the study, based on previous quantitative PCR assays, $(7,10)$ with focus on some of the most commonly non-inherited and non-shared alleles. Final primer and probe concentrations optimized for DQB1*04:02 and DQB1*06:02/03 at 100nM and 300nM, and DQB1*03:01 at 300nM and 100nM respectively. DNA was quantified using the QuantiFluor dsDNA system (Promega, Madison, USA). Ten wells were run for each sample, loaded at 10,000 genomic equivalent (gEq) equal to $33 \mathrm{ng}$; 100,000 gEq was screened per sample. MMc level was determined by total copy number of NIMA HLA allele specific positive cells per 1000000 host cells (as determined by beta-globin). A priori, we decided to consider a sample positive only if at least two of ten wells were positive, to minimize any potential false positives. Samples with only one positive well $(n=62)$ were set to an MMc value of zero.

As the measured MMc levels between probes are not directly comparable, we calculated MMc zscores by subtracting the NIMA specific mean and dividing by the standard deviation (both calculated from control children), all done on $\log _{2}$-transformed raw values.

\section{Other covariates}

A priori, we chose maternal smoking (at end of pregnancy), and child's sex, as our primary adjusting variables, as those variables have been associated with CD and could conceivably influence MMc. $(31,32)$ Maternal CD was only present in case children, and was therefore not included as an adjusting variable, but a sensitivity analysis was done where we excluded the dyads with maternal CD. As a sensitivity analysis, we also adjusted for other potential relevant variables as maternal age, pre-pregnancy BMI and caesarean section as these have in some studies been associated to offspring CD risk. Characteristics of the participants are presented in Table 1. 


\section{Statistical analysis}

We used logistic regression to estimate odds ratios (ORs) for childhood CD according to the presence of any MMc in cord blood (positive vs negative), and divided into three categories of quantities (negative, $\leq$ median and $>$ median of the quantifiable MMc values). We also tested whether the ranks of MMc differed between cases and controls using a Mann-Whitney U test. In a secondary analysis, we stratified the analyses by specific NIMAs. Due to missing data on covariates, not all participants were included in the adjusted analyses (number of participants included per analysis given in Table 2). To assess the sensitivity of our results towards the cutoff for MMc positivity, we reran the analysis when also calling samples positive if only one of 10 replicates were positive. We also investigated the potential association between HLA genotypes (either offspring or maternal HLA-DQ2.5, DQ8 or DQ6) and MMc positivity in the random control sample. All analyses were done in Stata Release 15 (College Station, Tx, USA).

\section{Results}

We measured MMc in 124 cases and 124 controls, and MMc was detected with similar frequencies in both groups (42 vs $43 \%$, respectively), resulting in an adjusted odds ratio (aOR) of $1.00(95 \%$ CI $0.59-1.70, \mathrm{p}=0.99)$ for the presence (detectable levels) of MMc in cord blood (Table 2). When analyzing the data as categories (divided into negative, $\leq$ median and $>$ median), no category reached statistical significance (aOR 1.08, 95\%CI $0.79-1.47, \mathrm{p}=0.63$ per increase in category; Table 2). The ranks of MMc values in cases and controls were also similar (MannWhitney $U$ test $\mathrm{p}=0.71$ ). The calculated MMc values are shown in Supplemental Figure 1 (Supplemental Digital Content, http://links.lww.com/MPG/B870). 
In a subgroup analysis, there was a possibly association between >median levels of $H L A$ $D Q B 1: 03 * 01 \mathrm{MMc}$ and later CD (aOR 3.12, 95\% CI 1.02 - 9.51, $\mathrm{p}=0.05$; Table 2). The ranks of this NIMA was significantly different between cases and controls (Wilcoxon rank-sum test $\mathrm{p}$ $=0.01)$. Presence of $H L A-D Q B 1: 03 * 01 \mathrm{MMc}$ in cord blood was not statistically associated with later development of $\mathrm{CD}$, although estimates were increased $(\mathrm{aOR} 2.38,95 \%$ CI $0.91-6.27, \mathrm{p}=$ 0.08; Table 2).

Running the analysis with samples positive only in one replicate set as positive, or additional statistical adjustment for maternal smoking, pre-pregnancy BMI, caesarean section, child's HLA genotype and sex (categorized as in Table 1) gave similar results as the main analysis (Table 3). Excluding dyads with maternal CD $(n=7)$ gave essentially unchanged results (data not shown). Neither offspring HLA genotype (DQ2.5 [aOR 1.0, 95\% CI 0.5-2.0, p=0.9], DQ8 [aOR 1.0, 95\% CI $0.7-1.6, \mathrm{p}=0.9$ ] or DQ6 [aOR 1.1, 95\% CI 0.4-3.0, $\mathrm{p}=0.8]$ ), or maternal HLA genotype (DQ2.5 [aOR 0.9, 95\% CI 0.4-2.4, p=0.9], DQ8 [aOR 1.2, 95\% CI 0.8-1.9, p=0.4] or DQ6 [aOR $1.2,95 \%$ CI $0.6-2.4, \mathrm{p}=0.7]$ ), was associated with MMc positivity.

\section{Discussion}

In this first study investigating MMc and $\mathrm{CD}$, we found no overall association between MMc in cord blood and CD.

Early life factors, which could play a significant role in the pathogenesis of autoimmune conditions have remained the least understood despite rigorous investigation. While MMc has been linked to autoimmunity, most studies are in patients after diagnosis, (6-11) and it is unclear whether MMc would be a risk factor for disease or involved in tissue repair. $(16,33,34)$ There are few studies of cord blood MMc and later disease. This is the first study on MMc and CD, and 
like our previous study on MMc and T1D in cord blood we do not find a clear association with later disease. (12) Taken together, this could indicate that MMc levels at birth are not predictive of later immune-mediated disease. Yet, we cannot rule out that MMc in specific tissues (e.g high amounts of MMc in the pancreas could be associated with T1D), specific MMc cell types, or at a later time could be associated with disease. It might also be that increased levels of MMc observed in diseases might be a result of maternal cells proliferating as a response to tissue damage, and thus therefore be a consequence of disease rather than a predisposing factor. Studies investigating MMc levels in patients with $\mathrm{CD}$ are necessary to investigate a potential later association between MMc and CD.

Strengths of this study include the prospective nature and large number of cord blood samples from the Norwegian population, Illumina genotyping on mothers and offspring for HLA imputation and identification of samples where the NIMA could be identified and tested for quantitatively. Prior to NIMA specific ddPCR analysis, all imputed HLA results were confirmed by conventional HLA analysis to prevent false negative results. The number of samples with detectable MMc levels are slightly higher or similar to what has been reported in other studies using cord blood, (35-38) which shows the suitability of sensitive PCR approaches such as the ddPCR method. Using samples taken at birth eliminates the risk that development of CD, or possible postnatal environmental factors risk factors influences our results.

A limitation is that although cord blood samples were available for 416 individuals who later developed CD, NIMA analysis was only possible on $124(\sim 30 \%)$ cases. This was due to lack of an informative NIMA in mother-child pairs, and individual ddPCR assays not being available for all HLA alleles. Although covering all possible NIMA alleles would be optimal, the benefit of establishing/optimizing new assays for every possible allele leads to limited returns on invested 
time and funds. As in any study reporting null findings, the possibility of a type 2 error should be considered. Our study could lack power due to the limited number of participants with a positive MMc measurement, but our study is also amongst the largest studies on MMc and disease $(8,34$, 39, 40). As this is an observational study, we cannot rule out unmeasured confounding. In general, participants in studies such as MoBa tend to be healthier, more educated and have higher socioeconomic status than the general population, which could make our results not generalizable. It seems implausible that MoBa participation or socioeconomic factors would be associated with MMc status. We cannot rule out that some controls would have unknown celiac disease, that some controls could develop CD after the study, or that some case children are misdiagnosed. Most case children in this cohort were diagnosed based on biopsy (83\%) and serology,(23) and we expect misdiagnosed cases to be rare. A plausible prevalence of misdiagnosed cases, or undiagnosed CD in control children (e.g in the range $0.5-2 \%$ ), would only lead to a couple of misclassified participants, and is unlikely to affect the interpretation of results substantially. It could also be that specific maternal cell types, genotypes, phenotypes or combinations of these, could be important in determining the influence from MMc in offspring. (5) Our main analysis was on general MMc levels at birth, and we only report subgroup analysis on the specific NIMAs used in the study. This is both due to lack of data (e.g on MMc cell types present), and to avoid multiple testing. The large number of potential subgroups and interactions that could be tested would probably lead to an unacceptable high number of false positive results due to multiple testing. We have available samples from birth, but transfer of maternal cells is believed to start from the second trimester, (41) and could fluctuate during gestation or due to labor. (42) 
In conclusion, our findings do not support an association between MMc levels at birth and later CD.

\section{Acknowledgements}

The Norwegian Mother, Father and Child Cohort Study is supported by the Norwegian Ministry of Health and Care Services and the Ministry of Education and Research. We are grateful to all the participating families in Norway who take part in this on-going cohort study. The Norwegian Childhood Diabetes Registry is funded by The South-Eastern Norway Regional Health Authority. We are thankful to the Norwegian Childhood Diabetes Registry and to the Norwegian Childhood Diabetes Study Group. Data from the Norwegian Patient Register has been used in this publication. The interpretation and reporting of these data are the sole responsibility of the authors, and no endorsement by the Norwegian patient register is intended nor should be inferred. SNP genotyping in this study was performed at the Genomics Core Facility, Norwegian Radium hospital, Oslo, Norway. 


\section{References}

1 Loubiere LS, Lambert NC, Flinn LJ, et al. Maternal microchimerism in healthy adults in lymphocytes, monocyte/macrophages and NK cells. Lab Invest 2006;86(11):1185-92.

2 Maloney S, Smith A, Furst DE, et al. Microchimerism of maternal origin persists into adult life. J Clin Invest 1999;104(1):41-7.

3 Mold JE, Michaelsson J, Burt TD, et al. Maternal alloantigens promote the development of tolerogenic fetal regulatory T cells in utero. Science 2008;322(5907):1562-5.

4 Kinder JM, Jiang TT, Ertelt JM, et al. Cross-Generational Reproductive Fitness Enforced by Microchimeric Maternal Cells. Cell 2015;162(3):505-15.

5 Leveque L, Hodgson S, Peyton S, et al. Selective organ specific inflammation in offspring harbouring microchimerism from strongly alloreactive mothers. J Autoimmun $2014 ; 50(51-8$.

6 Nelson JL Microchimerism and HLA relationships of pregnancy: implications for autoimmune diseases. Curr Rheumatol Rep 2001;3(3):222-9.

7 Nelson JL, Gillespie KM, Lambert NC, et al. Maternal microchimerism in peripheral blood in type 1 diabetes and pancreatic islet beta cell microchimerism. Proc Natl Acad Sci U S A 2007;104(5):1637-42.

8 Reed AM, Picornell YJ, Harwood A, et al. Chimerism in children with juvenile dermatomyositis. Lancet 2000;356(9248):2156-7.

9 Stevens AM, Hermes HM, Rutledge JC, et al. Myocardial-tissue-specific phenotype of maternal microchimerism in neonatal lupus congenital heart block. Lancet 2003;362(9396):1617-23. 
10 Lambert NC, Erickson TD, Yan Z, et al. Quantification of maternal microchimerism by HLA-specific real-time polymerase chain reaction: studies of healthy women and women with scleroderma. Arthritis Rheum 2004;50(3):906-14.

11 Khosrotehrani K, Guegan S, Fraitag S, et al. Presence of chimeric maternally derived keratinocytes in cutaneous inflammatory diseases of children: the example of pityriasis lichenoides. J Invest Dermatol 2006;126(2):345-8.

12 Tapia G, Mortimer G, Ye J, et al. Maternal microchimerism in cord blood and risk of childhood-onset type 1 diabetes. Pediatr Diabetes 2019;20(6):728-35.

13 Kurppa K, Laitinen A, Agardh D Coeliac disease in children with type 1 diabetes. Lancet Child Adolesc Health 2018;2(2):133-43.

14 Verdu EF, Danska JS Common ground: shared risk factors for type 1 diabetes and celiac disease. Nat Immunol 2018;19(7):685-95.

15 Karell K, Louka AS, Moodie SJ, et al. HLA types in celiac disease patients not carrying the DQA1*05-DQB1*02 (DQ2) heterodimer: results from the European Genetics Cluster on Celiac Disease. Hum Immunol 2003;64(4):469-77.

16 Nelson JL The otherness of self: microchimerism in health and disease. Trends Immunol 2012;33(8):421-7.

17 Hagopian W, Lee HS, Liu E, et al. Co-occurrence of Type 1 Diabetes and Celiac Disease Autoimmunity. Pediatrics 2017;140(5).

18 Liu E, Dong F, Baron AE, et al. High Incidence of Celiac Disease in a Long-term Study of Adolescents With Susceptibility Genotypes. Gastroenterology 2017;152(6):1329-36 e1. 
19 Mårild K, Ludvigsson JF, Størdal K Current evidence on whether perinatal risk factors influence coeliac disease is circumstantial. Acta Paediatr 2016;105(4):366-75.

20 Magnus P, Birke C, Vejrup K, et al. Cohort Profile Update: The Norwegian Mother and Child Cohort Study (MoBa). Int J Epidemiol 2016;45(2):382-8.

21 Rønningen KS, Paltiel L, Meltzer HM, et al. The biobank of the Norwegian Mother and Child Cohort Study: a resource for the next 100 years. Eur J Epidemiol 2006;21(8):61925.

22 Mårild K, Kahrs CR, Tapia G, et al. Infections and risk of celiac disease in childhood: a prospective nationwide cohort study. Am J Gastroenterol 2015;110(10):1475-84.

23 Emilsson L, Magnus MC, Stordal K Perinatal risk factors for development of celiac disease in children, based on the prospective Norwegian Mother and Child Cohort Study. Clin Gastroenterol Hepatol 2015;13(5):921-7.

24 Rothman K, Greenland S, Lash T. Case-control studies. In: K. Rothman, S. Greenland and T. Lash eds. Modern epidemiology. Philadelphia: Lippincott Williams \& Wilkins; 2008:111-27.

25 Størdal K, Mårild K, Tapia G, et al. Fetal and Maternal Genetic Variants Influencing Neonatal Vitamin D Status. J Clin Endocrinol Metab 2017;102(11):4072-79.

26 Paltiel L, Haugan A, Skjerden T, et al. The biobank of the Norwegian Mother and Child Cohort Study - present status. Nor J Epidemiol 2014;24(1-2):29-35.

27 Vistnes M, Tapia G, Mårild K, et al. Plasma immunological markers in pregnancy and cord blood: A possible link between macrophage chemo-attractants and risk of childhood type 1 diabetes. Am J Reprod Immunol 2018;79(3). 
28 Dilthey A, Leslie S, Moutsianas L, et al. Multi-population classical HLA type imputation. PLoS Comput Biol 2013;9(2):e1002877.

29 Aitken RJ, Mortimer GL, Gillespie KM Type 1 Diabetes High-Risk HLA Class II Determination by Polymerase Chain Reaction Sequence-Specific Primers. Methods Mol Biol 2016;1433(13-20.

30 Mårild K, Vistnes M, Tapia G, et al. Midpregnancy and cord blood immunologic biomarkers, HLA genotype, and pediatric celiac disease. J Allergy Clin Immunol 2017;139(5):1696-98.

31 Dixit R, Lebwohl B, Ludvigsson JF, et al. Celiac disease is diagnosed less frequently in young adult males. Dig Dis Sci 2014;59(7):1509-12.

32 Mårild K, Tapia G, Midttun Ø, et al. Smoking in pregnancy, cord blood cotinine and risk of celiac disease diagnosis in offspring. Eur J Epidemiol 2019;34(7):637-49.

33 Kinder JM, Stelzer IA, Arck PC, et al. Immunological implications of pregnancy-induced microchimerism. Nat Rev Immunol 2017;17(8):483-94.

34 Artlett CM, Sassi-Gaha S, Ramos RC, et al. Chimeric cells of maternal origin do not appear to be pathogenic in the juyenile idiopathic inflammatory myopathies or muscular dystrophy. Arthritis Res Ther 2015;17(238.

35 Hall JM, Lingenfelter P, Adams SL, et al. Detection of maternal cells in human umbilical cord blood using fluorescence in situ hybridization. Blood 1995;86(7):2829-32.

36 Lo YM, Lo ES, Watson N, et al. Two-way cell traffic between mother and fetus: biologic and clinical implications. Blood 1996;88(11):4390-5. 
37 Roh EY, Yoon JH, Shin S, et al. Frequency of fetal-maternal microchimerism: an analysis of the HLA-DRB1 gene in cord blood and maternal sample pairs. J Matern Fetal Neonatal Med 2017;30(21):2613-19.

38 Scaradavou A, Carrier C, Mollen N, et al. Detection of maternal DNA in placental/umbilical cord blood by locus-specific amplification of the noninherited maternal HLA gene. Blood 1996;88(4):1494-500.

39 Harrington WE, Kanaan SB, Muehlenbachs A, et al. Maternal Microchimerism Predicts Increased Infection but Decreased Disease due to Plasmodium falciparum During Early Childhood. J Infect Dis 2017;215(9):1445-51.

40 Ye J, Vives-Pi M, Gillespie KM Maternal microchimerism: increased in the insulin positive compartment of type 1 diabetes pancreas but not in infiltrating immune cells or replicating islet cells. PLoS One 2014;9(1):e86985.

41 Jonsson AM, Uzunel M, Gotherstrom C, et al. Maternal microchimerism in human fetal tissues. Am J Obstet Gynecol 2008;198(3):325 e1-6.

42 Masuzaki H, Miura K, Miura S, et al. Labor increases maternal DNA contamination in cord blood. Clin Chem 2004;50(9):1709-11. 


\section{Figure 1 legend}

Figure 1 shows the study design, and distribution of NIMAs measured. Panel A is an illustration of informative vs. non-informative NIMA. A non-informative genotype results if the mother is homozygous, or if mother and child share both HLA-DQB1 alleles, which masks the NIMA. Panel B is a Sankey diagram with participant numbers at each category in this study, and how many cases and controls were included in the final analysis (generated using R (version 3.6.1), riverplot package (version 0.6, Weiner, J. 2017)).

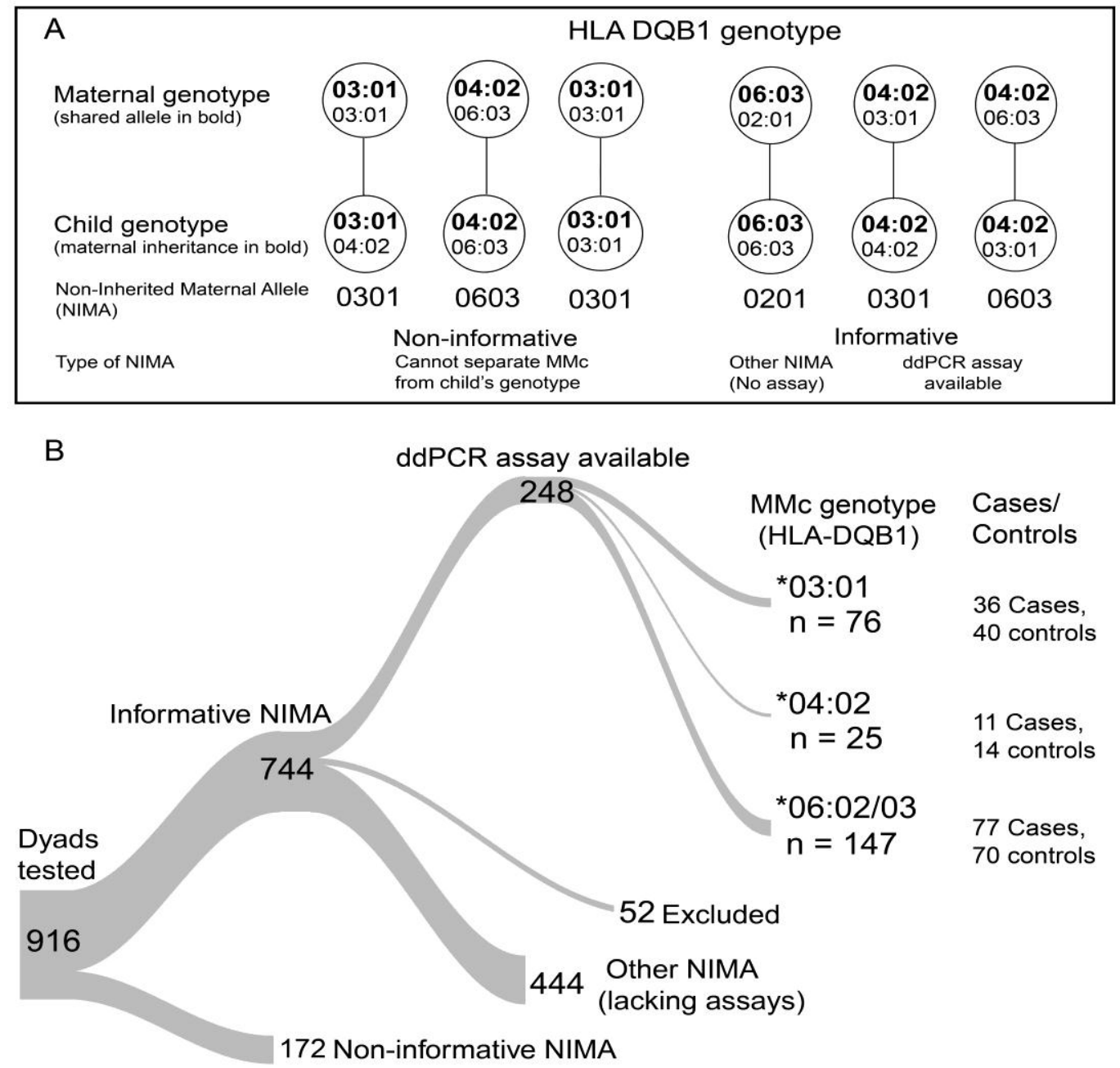


Table 1: Characteristics of cases with celiac disease diagnosis and randomly selected controls in the present study.

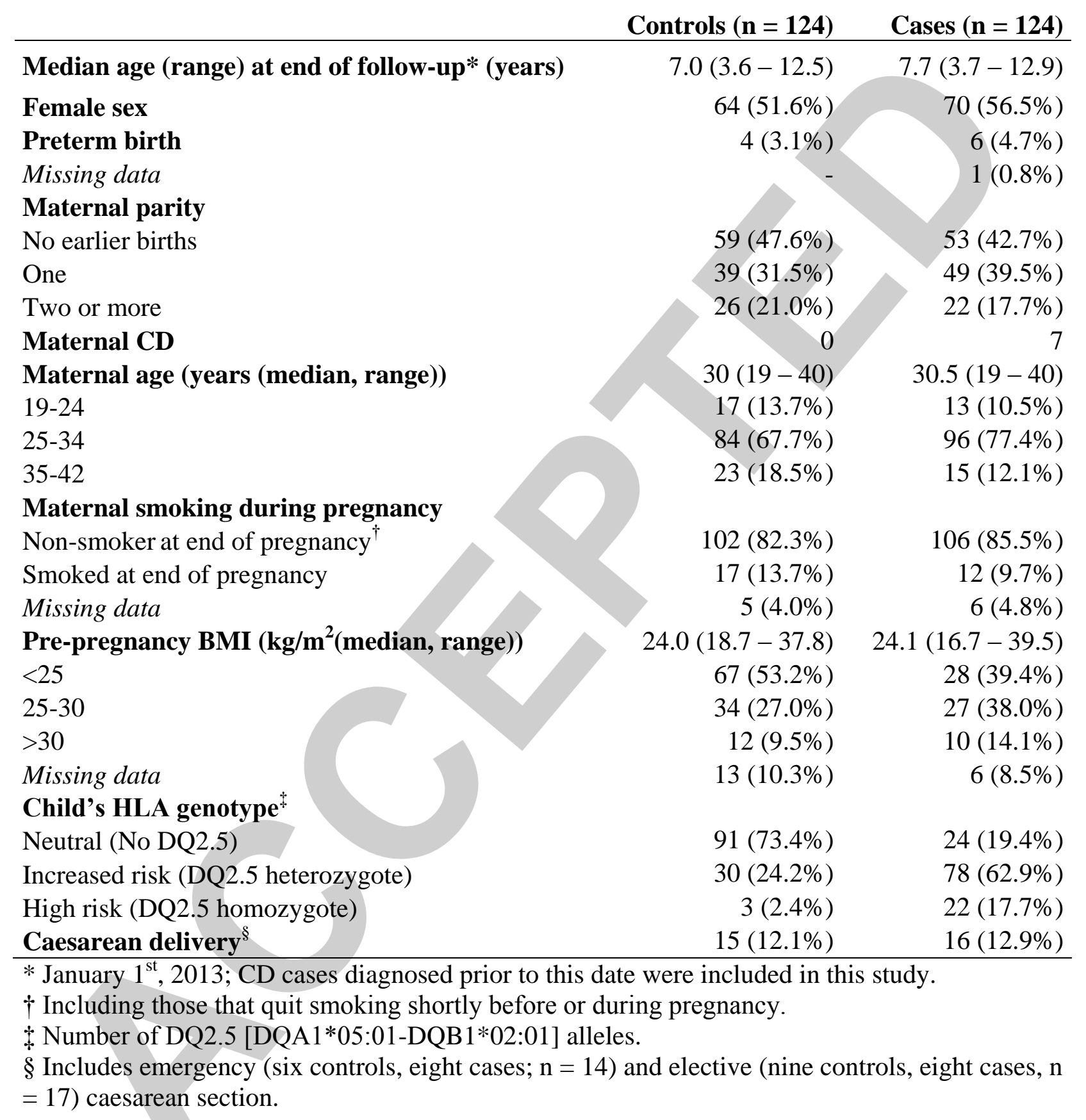


Table 2: Odds ratios for the association between MMc, measured by non-inherited maternal HLA alleles, in cord blood and offspring celiac disease.

\begin{tabular}{|c|c|c|c|c|c|}
\hline Presence of detectable MMc & Cases* & Controls* & OR $(95 \%$ CI $)$ & $\begin{array}{l}\text { aOR }(95 \% \\
\text { CI })^{\dagger}\end{array}$ & $\begin{array}{c}\text { P- } \\
\text { value }^{\dagger}\end{array}$ \\
\hline Any $^{\ddagger}$ NIMA & $49 / 118$ & $50 / 119$ & $\begin{array}{r}0.97(0.58- \\
1.60)\end{array}$ & $\begin{array}{r}1.00(0.59- \\
1.70)\end{array}$ & 0.99 \\
\hline DQB1*03:01 NIMA & $24 / 35$ & $18 / 40$ & $\begin{array}{r}2.78(1.08- \\
7.14)\end{array}$ & $\begin{array}{r}2.38(0.91- \\
6.27)\end{array}$ & 0.08 \\
\hline DQB1*06:02/03 NIMA & $22 / 72$ & $28 / 65$ & $\begin{array}{r}0.57(0.29- \\
1.12)\end{array}$ & $\begin{array}{r}0.65(0.32- \\
1.34)\end{array}$ & 0.25 \\
\hline \multicolumn{6}{|l|}{ Categorical analysis $^{\S}$} \\
\hline Any ${ }^{*}$ NIMA, Undetected & $69 / 118$ & $69 / 119$ & Ref. & Ref. & \\
\hline Any ${ }^{*}$ NIMA $\leq$ median & $19 / 118$ & $26 / 119$ & $\begin{array}{r}0.72(0.37- \\
1.42)\end{array}$ & $\begin{array}{r}0.76(0.38- \\
1.52)\end{array}$ & 0.44 \\
\hline Any ${ }^{*}$ NIMA $>$ median & $30 / 118$ & $24 / 119$ & $\begin{array}{r}1.21(0.66- \\
2.21)\end{array}$ & $\begin{array}{r}1.25(0.66- \\
2.36)\end{array}$ & 0.50 \\
\hline Per category increase & $49 / 118$ & $50 / 119$ & $\begin{array}{r}1.06(0.79- \\
1.42)\end{array}$ & $\begin{array}{r}1.08(0.79- \\
1.47)\end{array}$ & 0.63 \\
\hline DQB1*03:01 NIMA, undetected & $11 / 35$ & $22 / 40$ & Ref. & Ref. & \\
\hline DQB1*03:01 NIMA $\leq$ median & $8 / 35$ & $9 / 40$ & $\begin{array}{r}1.78(0.54- \\
5.88)\end{array}$ & $\begin{array}{r}1.64(0.48- \\
5.53)\end{array}$ & 0.43 \\
\hline DQB1*03:01 NIMA > median & $16 / 35$ & & $\begin{array}{r}3.78(1.28- \\
11.18)\end{array}$ & $\begin{array}{r}3.12(1.02- \\
9.51)\end{array}$ & 0.05 \\
\hline Per category increase & $24 / 35$ & $18 / 40$ & $\begin{array}{r}1.94(1.13- \\
3.33)\end{array}$ & $\begin{array}{r}1.76(1.01- \\
3.07)\end{array}$ & 0.05 \\
\hline $\begin{array}{l}\text { DQB } 1 * 06: 02 / 03 \text { NIMA, } \\
\text { undetected }\end{array}$ & $50 / 72$ & $37 / 65$ & Ref. & Ref. & \\
\hline DQB1*06:02/03 NIMA $\leq$ median & $11 / 72$ & $15 / 65$ & $\begin{array}{r}0.54(0.22- \\
1.30)\end{array}$ & $\begin{array}{r}0.70(0.28- \\
1.76)\end{array}$ & 0.45 \\
\hline DQB1*06:02/03 NIMA > median & $11 / 72$ & $13 / 65$ & $\begin{array}{r}0.60(0.26- \\
1.39)\end{array}$ & $\begin{array}{r}0.61(0.24- \\
1.54)\end{array}$ & 0.30 \\
\hline Per category increase & $22 / 72$ & $28 / 65$ & $\begin{array}{r}0.75(0.50- \\
1.12)\end{array}$ & $\begin{array}{r}0.78(0.51- \\
1.21)\end{array}$ & 0.27 \\
\hline
\end{tabular}

OR: Odds Ratio; aOR: adjusted OR; CI: Confidence Interval

* positive / total number of participants included in the adjusted analysis. There were 124 cases and 124 controls in total in the unadjusted analyses. Numbers vary due to missing values for some covariates (see Table 1). The results are shown for the whole group (the first line, Any NIMA), followed by subgroups analyses. $\dagger$ adjusted for maternal smoking and child's sex $\ddagger$ combining DQB1*03:01, *04:02 and *06:02/03. The DQB1*04:02 NIMA subgroup was not analyzed separately due to low number of children $(n=25)$ with measurable quantities $(n=7)$ in this group.

$\S$ the median value was calculated from controls with detectable levels 


\begin{tabular}{|c|c|c|c|}
\hline & $\begin{array}{l}\text { aOR }(95 \% \mathrm{CI})^{*}, \\
\text { Main analysis }\end{array}$ & $\begin{array}{c}\mathrm{aOR}(95 \% \mathrm{CI})^{\dagger}, \\
\text { additionally adjusted }\end{array}$ & $\begin{array}{c}\text { aOR }(95 \% \mathrm{CI})^{*}, \\
\text { including unreplicated } \\
\text { positives }\end{array}$ \\
\hline \multicolumn{4}{|l|}{ Presence of detectable MMc } \\
\hline Any ${ }^{\ddagger}$ NIMA & $1.00(0.59-1.70)$ & $0.72(0.37-1.40)$ & $1.27(0.74-2.18)$ \\
\hline DQB1*03:01 NIMA & $2.38(0.91-6.27)$ & $2.71(0.63-11.62)$ & $2.43(0.79-7.46)$ \\
\hline DQB1*06:02/03 NIMA & $0.65(0.32-1.34)$ & $0.46(0.19-1.12)$ & $0.89(0.43-1.82)$ \\
\hline \multicolumn{4}{|l|}{ Categorical analysis $^{\S}$} \\
\hline Any ${ }^{\ddagger}$ NIMA, Undetected & Ref. & Ref. & Ref. \\
\hline Any ${ }^{\ddagger}$ NIMA $\leq$ median & $0.76(0.38-1.52)$ & $0.51(0.21-1.24)$ & $0.91(0.47-1.77)$ \\
\hline Any ${ }^{\ddagger}$ NIMA $>$ median & $1.25(0.66-2.36)$ & $0.94(0.43-2.09)$ & $1.61(0.88-2.97)$ \\
\hline Per category increase & $1.08(0.79-1.47)$ & $0.92(0.62-1.35)$ & $1.27(0.94-1.73)$ \\
\hline DQB1*03:01 NIMA, undetected & Ref. & Ref. & Ref. \\
\hline DQB1*03:01 NIMA $\leq$ median & $1.64(0.48-5.53)$ & $0.93(0.13-6.87)$ & $0.86(0.20-3.62)$ \\
\hline DQB1*03:01 NIMA > median & $3.12(1.02-9.51)$ & $4.82(0.88-26.39)$ & $3.94(1.19-13.04)$ \\
\hline Per category increase & $1.76(1.01-3.07)$ & $2.15(0.94-4.96)$ & $2.12(1.16-3.86)$ \\
\hline $\begin{array}{l}\text { DQB1*06:02/03 NIMA, } \\
\text { undetected }\end{array}$ & & Ref. & Ref. \\
\hline DQB $1 * 06: 02 / 03$ NIMA $\leq$ median & $0.70(0.28-1.76)$ & $0.47(0.15-1.51)$ & $0.99(0.43-2.29)$ \\
\hline DQB $1 * 06: 02 / 03$ NIMA > median & $0.61(0.24-1.54)$ & $0.46(0.15-1.38)$ & $0.79(0.34-1.84)$ \\
\hline Per category increase & $0.78(0.51-1.21)$ & $0.63(0.38-1.07)$ & $0.89(0.58-1.36)$ \\
\hline
\end{tabular}

aOR: adjusted OR; CI: Confidence Interval.

Main analysis is shown leftmost for comparison, followed by an analysis including more covariates, and an analysis including the measured values of samples which were positive in only one of ten replicates (originally set to zero in the main analysis).

* adjusted for maternal smoking and child's sex

$\dagger$ adjusted for maternal age, pre-pregnancy BMI, maternal smoking, caesarean section, child's sex and HLA risk for $\mathrm{CD}(0,1$ or $2 \mathrm{DQ} 2.5$ alleles).

$\ddagger$ combining DQB1*03:01, *04:02 and *06:02/03. The DQB1*04:02 NIMA subgroup was not analysed separately due to low number of children with measurable quantities in this group. $\S$ the median value was calculated from controls with detectable levels. 